\title{
Leitura de rótulos de alimentos por frequentadores de um estabelecimento comercial
}

\author{
Reading of food labels by commercial establishment shoppers
}

\author{
Bruna Marzarotto ${ }^{a}$, Márcia Keller Alves ${ }^{b}$ \\ a Nutricionista. Pós-graduanda em Gestão da Qualidade e Controle Higiênico Sanitária na Produção de Alimentos da Faculdade Nossa Senhora de Fátima. \\ b Nutricionista. Mestre em Biologia Celular e Molecular. Docente e Coordenadora do Núcleo de Esstudos e Pesquisa em Alimentos e Nutrição da \\ Faculdade Nossa Senhora de Fátima.
}

\begin{tabular}{l|l} 
RESUMO Objetivo: Avaliar se os frequentadores de um estabelecimento comercial têm o hábito de ler e se conseguem &
\end{tabular} compreender a rotulagem nutricional dos alimentos.

Materiais e Métodos: Estudo transversal, realizado em 2012 e 2013, com 227 indivíduos selecionados e entrevistados aleatoriamente em um estabelecimento comercial alimentício de Caxias do Sul-RS. O questionário aplicado avaliou o nível socioeconômico, hábito e motivo de leitura, principal informação observada, critérios de escolha dos alimentos, entre outros. A associação entre as variáveis foi analisada através do teste qui-quadrado, utilizando o software SPSS versão 19.0.

Resultados: A amostra foi formada principalmente por consumidores do gênero feminino, casados, com menos de 30 anos, com ensino superior completo e renda mensal acima de R\$1.500,00. A maioria (70\%) possui o hábito de leitura e 40,5\% o fazem no supermercado. A informação nutricional mais lida é o valor calórico, motivada pela busca por uma alimentação saudável. A internet é a fonte de informação mais utilizada para a busca de conhecimentos sobre alimentação e nutrição. Qualidade e sabor do alimento são os critérios mais destacados pelos clientes na hora da compra do alimento. Entre os entrevistados, 39,2\% diz ter 30 minutos para a realização das compras, tempo suficiente para efetuar a leitura dos rótulos. Os indivíduos dizem utilizar as informações encontradas para manter uma alimentação saudável, porém, não acreditam conhecer o suficiente de nutrição.

Conclusão: O hábito de leitura está presente entre os entrevistados, que utilizavam a declaração nutricional para orientação alimentar com o objetivo de manter uma dieta saudável. Entretanto, os participantes não acreditam ter conhecimento suficiente de nutrição para a compreensão das informações contidas nos rótulos.

Palavras-chave: rotulagem de alimentos; informação nutricional; hábitos alimentares.

Objective: To assess whether the consumers of a local business have the habit of reading and are able to understand the nutritional labeling of food.

Material and Methods: Cross-sectional study carried out from 2012 to 2013, with 227 individuals interviewed randomly in a food business establishment at Caxias do Sul-RS. The questionnaire assessed the socioeconomic level, habit and reason for reading, main observed information, food selection criteria, among others. The association between variables was analyzed through the chi-square test, using SPSS software version 19.0.

Results: The sample was made up mainly of female consumers, married, with less than 30 years, with higher education and monthly income above $R \$$ 1.500.00. The majority are used to reading and doing it at the supermarket. The most read nutrition information is the caloric value, motivated by the search for a healthier diet. The internet is the most used source of information in the searching for knowledge about food and nutrition. Quality and food taste are the most outstanding criteria by customers when buying food. Among the respondents, $39.2 \%$ said to have 30 minutes to carry out the purchases and that it would be enough to read the labels. Individuals showed to use this information for maintaining a healthy diet; however, they do not believe to know enough about nutrition.

Conclusion: The habit of reading is present among the interviewed people, who use the nutrition information as a food guidance in order to maintain a healthy diet. However, the participants do not believe that they have enough knowledge of nutrition for understanding the nutritional labeling of food.

Keywords: food labeling; nutritional facts; food habits. 


\section{INTRODUÇÃO}

As mudanças nos hábitos alimentares das pessoas vêm fazendo com que haja uma maior busca por alimentos prontos e de fácil preparo, elevando o consumo de gorduras totais, carboidratos simples, colesterol e calorias, reduzindo os alimentos ricos em fibras, vitaminas, minerais e com baixo teor de ácidos graxos insaturados ${ }^{1,2}$. Este consumo alimentar é um determinante da saúde, cujo caráter positivo ou negativo depende de informações adequadas, sendo de fundamental importância intervenções de educação nutricional que auxiliem a população na escolha de alimentos mais saudáveis ${ }^{3}$.

A rotulagem nutricional é muito importante quando se trata de escolhas alimentares saudáveis, pois é no rótulo do alimento que são fornecidas aos consumidores as informações necessárias para comparação e esclarecimento, e para que ele possa decidir pela compra do produto ${ }^{4}$. Entende-se por rotulagem nutricional toda inscrição destinada a informar ao consumidor sobre as propriedades nutricionais de um alimento, a qual deve compreender a declaração do valor energética e nutriente e a declaração das propriedades nutricionais (informação nutricional complementar $)^{5}$.

Para que o consumidor possa utilizar esta ferramenta é preciso que a veracidade das informações apresentadas pelo rótulo nutricional em alimentos seja garantida, cumprindo assim o objetivo de auxiliar ao consumidor em suas escolhas e aos profissionais de saúde, na orientação para a composição da dieta $^{6}$. Diversos estudos apontam não conformidades entre dados descritos na rotulagem nutricional e a real composição do alimento fazendo com que o consumidor se sinta enganado ${ }^{6-13}$.

A legislação referente a informação nutricional constitui um importante avanço na manutenção e proteção à saúde do consumidor ${ }^{14}$. Contudo, um reduzido nível de entendimento a respeito do significado e aplicação da informação nutricional por grande parte dos consumidores, aponta para a necessidade de repensar a forma de apresentação das informações contidas no rótulo dos alimentos, bem como a postura dos órgãos responsáveis pelo atendimento aos consumidores. Por isso, o objetivo deste trabalho é avaliar o hábito de leitura e a compreensão de rótulos de alimentos por frequentadores de um estabelecimento comercial do ramo alimentício de Caxias do Sul-RS.

\section{MATERIAIS E MÉTODOS}

A pesquisa foi realizada no período de novembro de 2012 a março de 2013, após a aprovação do Comitê de Ética em Pesquisa (CEP) da Faculdade Nossa Senhora de
Fátima sob número de parecer 0360401123-11, observando todos os dispostos acerca da ética em pesquisa com seres humanos. Desta forma, os participantes foram preservados pelo Termo de Consentimento Livre e Esclarecido (TCLE), o qual continha informações sobre o estudo e seus objetivos. Este foi assinado em duas vias, sendo que uma ficou em posse do entrevistado e outra do entrevistador; a cópia que ficou com o entrevistador será arquivada durante cinco anos pelo pesquisador responsável da pesquisa e, após este período, será incinerada.

Os dados foram coletados em um estabelecimento comercial do ramo de hortifrutí da cidade da Caxias do Sul-RS. Os entrevistados foram escolhidos aleatoriamente enquanto faziam suas compras, sendo os critérios de inclusão para participar do estudo ter idade igual ou superior a 18 anos e ser alfabetizado; foram excluídos os indivíduos que não responderam a todas as perguntas do questionário. Para a coleta dos dados foi utilizado um questionário estruturado validado ${ }^{15}$. Todos responderam treze questões que incluíam dados sobre o nível socioeconômico e demográfico (variáveis sexo, idade, estado civil, escolaridade e renda familiar) e sobre comportamento do consumidor frente à utilização de rotulagem nutricional (variáveis hábito, motivo que os levam a realizar a leitura da rotulagem nutricional, que tipo de informações buscam nestes rótulos, fontes de informação sobre alimentação e nutrição e qual o grau de importância de alguns itens no ato da compra dos produtos). A coleta de dados foi realizada pela pesquisadora em horário comercial, durante a semana e aos finais de semana.

A análise estatística foi realizada no software Statistical Package for the Social Sciences ${ }^{\circledR}$ (SPSS) para Windows ${ }^{\circledR}$, versão 19.0. O teste qui-quadrado de Pearson foi utilizado para testar a associação entre as seguintes variáveis: gênero, faixa etária, estado civil, escolaridade, renda e hábito de leitura dos rótulos dos alimentos. Os resultados foram descritos através de frequência relativa (\%) e absoluta (n) e o nível de significância na associação foi $p<0,05$.

\section{RESULTADOS}

Foram entrevistados 227 consumidores frequentadores de um estabelecimento comercial da área de hortifrutí de Caxias do Sul-RS. As características da população estudada estão apresentadas na Tabela 1. Predominantemente, a população foi constituída de indivíduos do gênero feminino, com idade entre 19 e 29 anos, casados, com ensino superior completo e que tem como renda mais de $\mathrm{R} \$ 1.500,00$ mensais.

Quanto ao hábito de leitura da rotulagem, o perfil dos sujeitos de pesquisa pode ser visualizado na Tabela 2. A maioria dos entrevistados possui o hábito de ler a rotulagem 
Tabela 1. Características da amostra de frequentadores de um estabelecimento comercial do ramo alimentício.

\begin{tabular}{|c|c|c|}
\hline & $\begin{array}{l}\text { Frequência } \\
\text { (n) }\end{array}$ & $\begin{array}{l}\text { Percentual } \\
(\%)\end{array}$ \\
\hline \multicolumn{3}{|l|}{ Sexo } \\
\hline Feminino & 180 & 79,3 \\
\hline Masculino & 47 & 20,7 \\
\hline \multicolumn{3}{|l|}{ Faixa Etária (em anos) } \\
\hline $19-29$ & 79 & 34,8 \\
\hline $30-39$ & 58 & 25,6 \\
\hline $40-49$ & 44 & 19,4 \\
\hline$>50$ anos & 46 & 20,3 \\
\hline \multicolumn{3}{|l|}{ Estado Civil } \\
\hline Solteiro & 80 & 35,2 \\
\hline Casado & 136 & 59,9 \\
\hline Divorciado & 6 & 2,6 \\
\hline Viúvo & 5 & 2,2 \\
\hline \multicolumn{3}{|l|}{ Escolaridade } \\
\hline Ensino Fundamental Completo & 29 & 12,8 \\
\hline Ensino Médio Completo & 76 & 33,5 \\
\hline Ensino Superior Completo & 122 & 53,7 \\
\hline \multicolumn{3}{|l|}{ Renda (em reais $-\mathrm{R} \$$ ) } \\
\hline Até 300 & 11 & 4,8 \\
\hline 300 a 900 & 24 & 10,6 \\
\hline 900 a 1500 & 54 & 23,8 \\
\hline$>1500$ & 138 & 60,8 \\
\hline
\end{tabular}

Tabela 2. Hábitos de leitura da rotulagem nutricional por frequentadores de um estabelecimento comercial do ramo alimentício.

\begin{tabular}{lcc}
\hline & $\begin{array}{c}\text { Frequência } \\
(\mathbf{n})\end{array}$ & $\begin{array}{c}\text { Percentual } \\
(\mathbf{\%})\end{array}$ \\
\hline $\begin{array}{l}\text { Hábito de ler os rótulos } \\
\text { Sim }\end{array}$ & 158 & 69,6 \\
Não & 69 & 30,4 \\
Local em que lê & 54 & \\
Não Le & 92 & 23,8 \\
No supermercado & 36 & 15,9 \\
$\quad$ Em casa & 45 & 19,8 \\
$\quad$ No supermercado e em casa & & \\
Motivo de ler & 54 & 23,8 \\
Não Lê & 21 & 9,3 \\
Busca a redução do peso corpóreo & 117 & 51,1 \\
Busca uma alimentação saudável & 7 & 3,1 \\
Tem diagnóstico de colesterol elevado & 5 & 2,2 \\
Tem diagnóstico de hipertensão & 23 & 10,1 \\
\hline Outro motivo & & \\
\hline
\end{tabular}

nutricional e o faz no supermercado. O motivo que os leva a conhecer as informações nutricionais no rótulo do alimento é predominantemente a busca por uma alimentação saudável.

As informações nutricionais mais procuradas pelos consumidores estão descritas na Tabela 3. Destaca-se predominantemente a procura pelo valor de calorias nos alimentos consumidos, seguidos por sódio e gorduras. Por outro lado, uma parte dos entrevistados respondeu que não lê as informações nutricionais.

A fonte de informação predominantemente utilizada pelos sujeitos está descrita na Tabela 4. Os entrevistados utilizam principalmente a internet para buscar informações sobre alimentação e nutrição. Em segundo lugar, está o profissional de Nutrição.

O grau de importância que os sujeitos atribuem aos critérios qualidade, sabor, preço, marca e embalagem durante a compra do alimento está descrito na Tabela 5. Os critérios qualidade e sabor destacaram-se como muito importante.

Tabela 3. Elementos nutricionais consultados na declaração nutricional dos rótulos dos alimentos por frequentadores de um estabelecimento comercial do ramo alimentício.

\begin{tabular}{lcc}
\hline \multicolumn{1}{|c}{ Informação nutricional que procura } & $\begin{array}{c}\text { Frequência } \\
(\mathbf{n})\end{array}$ & $\begin{array}{c}\text { Percentual } \\
\text { (\%) }\end{array}$ \\
\hline Valor calórico & 63 & 27,8 \\
Gorduras & 33 & 14,5 \\
Colesterol & 10 & 4,4 \\
Fibras & 6 & 2,6 \\
Proteínas & 7 & 3,1 \\
Cálcio & 2 & 0,9 \\
Açúcar & 11 & 4,8 \\
Sódio & 34 & 15 \\
Vitamina C & 6 & 2,6 \\
Outros & 11 & 4,8 \\
Não lê & 44 & 19,4 \\
\hline
\end{tabular}

Tabela 4. Fonte de informação utilizada para obter conhecimentos sobre alimentação e nutrição por frequentadores de um estabelecimento comercial do ramo alimentício.

\begin{tabular}{lcc}
\hline \multicolumn{1}{|c}{ Fonte de informação } & $\begin{array}{c}\text { Frequência } \\
(\mathbf{n})\end{array}$ & $\begin{array}{c}\text { Percentual } \\
(\mathbf{\%})\end{array}$ \\
\hline Livros, revistas e jornais & 36 & 15,9 \\
Televisão & 25 & 11 \\
Amigos/família & 15 & 6,6 \\
Médico & 13 & 5,7 \\
Nutricionista & 57 & 25,1 \\
Embalagem & 4 & 1,8 \\
Internet & 72 & 31,7 \\
Outro & 5 & 2,2 \\
\hline
\end{tabular}


Tabela 5. Grau de importância atribuída aos critérios de qualidade dos produtos alimentícios por frequentadores de um estabelecimento comercial do ramo alimentício.

\begin{tabular}{|c|c|c|}
\hline Grau de importância & $\begin{array}{l}\text { Frequência } \\
\text { (n) }\end{array}$ & $\begin{array}{c}\text { Percentual } \\
(\%)\end{array}$ \\
\hline \multicolumn{3}{|l|}{ Qualidade } \\
\hline Pouca importância & 5 & 2,2 \\
\hline Importante & 68 & 30,0 \\
\hline Muito importante & 154 & 67,8 \\
\hline \multicolumn{3}{|l|}{ Sabor } \\
\hline Pouca importância & 9 & 3,9 \\
\hline Importante & 86 & 37,9 \\
\hline Muito importante & 132 & 58,1 \\
\hline \multicolumn{3}{|l|}{ Valor nutricional } \\
\hline Pouca importância & 65 & 19 \\
\hline Importante & 106 & 46,7 \\
\hline Muito importante & 76 & 33,5 \\
\hline \multicolumn{3}{|l|}{ Preço } \\
\hline Pouca importância & 44 & 19,4 \\
\hline Importante & 125 & 55,1 \\
\hline Muito importante & 58 & 25,6 \\
\hline \multicolumn{3}{|l|}{ Marca } \\
\hline Pouca importância & 119 & 52,4 \\
\hline Importante & 84 & 37,0 \\
\hline Muito importante & 24 & 10,6 \\
\hline \multicolumn{3}{|l|}{ Embalagem } \\
\hline Pouca importância & 81 & 35,7 \\
\hline Importante & 93 & 41,0 \\
\hline Muito importante & 53 & 23,3 \\
\hline
\end{tabular}

O valor nutricional dos alimentos, o preço e a embalagem destacaram-se como sendo importantes no ato da compra. E a marca do produto destacou-se como sendo de pouca importância para a decisão de compra ou não do produto.

A rotina dos entrevistados em relação à leitura dos rótulos dos alimentos está descrita na Tabela 6. Destacase que grande parte dos indivíduos têm 30 minutos para realizar suas compras durante o dia, e que neste tempo conseguem ler os rótulos dos alimentos. A maioria respondeu que utiliza as informações encontradas nos rótulos para uma dieta saudável e que não acreditam ter conhecimento suficiente de nutrição para a compreensão das mesmas, mesmo encontrando as informações nutricionais em todos os alimentos que adquirem.

Houve diferença estatística quanto à prevalência de leitura entre os gêneros $(p=0,02)$, mostrando que as mulheres leem os rótulos significativamente mais do que os homens, conforme descrito na Tabela 7. Houve ainda diferença significativa em relação à idade $(p=0,01)$, escolaridade $(p=0,004)$ e renda $(p=0,003)$, mostrando associação linear entre estas variáveis e o hábito de leitura.
Tabela 6. Rotina diária dos consumidores em relação à compra de alimentos, leitura e compreensão dos rótulos dos alimentos por frequentadores de um estabelecimento comercial do ramo alimentício.

\begin{tabular}{lcc}
\hline \multicolumn{1}{|c}{ Rotina diária dos sujeitos } & $\begin{array}{c}\text { Frequência } \\
(\mathbf{n})\end{array}$ & $\begin{array}{c}\text { Percentual } \\
(\%)\end{array}$ \\
\hline Quanto tempo para realizar compras & 33 & 14,5 \\
$\quad 15$ minutos & 89 & 39,2 \\
30 minutos & 67 & 29,5 \\
$\quad 1$ hora & 38 & 16,7 \\
$\quad$ Mais de 1 hora & & \\
Tempo para ler rótulos durante as compras & 121 & 53,3 \\
$\quad$ Sim & 106 & 46,7 \\
$\quad$ Não & & \\
Utiliza informações para manter uma & & \\
dieta saudável & 146 & 64,3 \\
$\quad$ Sim & 81 & 35,7 \\
$\quad$ Não & & \\
Conhece suficiente de nutrição para & & \\
utilizar a rotulagem nutricional & 95 & 41,9 \\
$\quad$ Sim & 132 & 58,1 \\
$\quad$ Não & & \\
Encontra as informações nutricionais \\
nos rótulos \\
$\quad$ Sim \\
Não
\end{tabular}

Tabela 7. Associação entre as variáveis e o hábito de leitura por frequentadores de um estabelecimento comercial do ramo alimentício.

\begin{tabular}{|c|c|c|}
\hline Demográficas & $\begin{array}{c}\text { Prevalência de } \\
\text { leitura (\%) }\end{array}$ & Valor de $p$ \\
\hline Gênero* & & 0,002 \\
\hline Feminino & 74,4 & \\
\hline Masculino & 51,1 & \\
\hline Faixa Etária** & & 0,010 \\
\hline $18-29$ & 59,5 & \\
\hline $30-39$ & 69,0 & \\
\hline $40-49$ & 88,6 & \\
\hline$>50$ anos & 69,6 & \\
\hline Estado Civil & & 0,689 \\
\hline Solteiro & 65,0 & \\
\hline Casado & 72,1 & \\
\hline Divorciado & 66,7 & \\
\hline Viúvo & 80,0 & \\
\hline Escolaridade** & & 0,004 \\
\hline Ensino Fundamental & 48,3 & \\
\hline Ensino Médio & 67,1 & \\
\hline Ensino Superior & 76,2 & \\
\hline Renda** & & 0,003 \\
\hline Até 300 & 31,8 & \\
\hline 300 a 900 & 41,7 & \\
\hline 900 a 1500 & 53,7 & \\
\hline$>1500$ & 79,7 & \\
\hline
\end{tabular}

* Chi quadrado de Pearson; **Associação linear. 


\section{DISCUSSÃO}

Os resultados descritivos do presente estudo estão de acordo com outros estudos ${ }^{3,15-17}$ nos quais a amostra é formada principalmente por mulheres casadas e jovens, que são responsáveis pelas compras da casa, com um poder aquisitivo maior (avaliado através da renda mensal) e alto grau de instrução (ensino superior completo).

No presente estudo, os consumidores alegaram ter o hábito de ler a rotulagem nutricional dos alimentos, o que corrobora com dados de outras pesquisas $3,16,18$, que demonstram que o hábito da leitura dos rótulos dos alimentos está presente em $81 \%$ a $94,6 \%$ dos entrevistados, os quais consultam os rótulos nas suas aquisições de alimentos, sendo $52 \%$ leitores constantes e $28,7 \%$ não constantes.

Entre a população entrevistada, grande parte relatou buscar a informação nutricional antes da finalização da compra do produto, ainda no supermercado. Este resultado opõe-se a outro estudo ${ }^{15}$, em que apenas $23 \%$ dos entrevistados dizem ler as informações nutricionais nos supermercados e que $45,5 \%$ leem a rotulagem nutricional apenas em casa. Estes resultados demonstram a importância da criação de medidas que incentivem a leitura da rotulagem nutricional, buscando informações sobre o alimento que vai consumir, pois a prática da leitura dos rótulos nos alimentos deveria ser adotada para a escolha do melhor produto que convém ao consumidor ${ }^{19}$.

A rotulagem dos alimentos tem caráter informativo, e, deste modo, a prática da leitura deveria acontecer no momento da compra do alimento ${ }^{20}$. Neste contexto, o tempo de permanência no supermercado, menor quando comprado a outro estudo ${ }^{16}$, pode influenciar na leitura na hora da compra. No entanto, na maioria das vezes, a grande dificuldade dos consumidores é a falta de compreensão dos termos utilizados, ocasionalmente muito técnicos. O autor relata ainda que há uma contradição quanto à finalidade dos rótulos dos alimentos: por um lado os mesmos representam um elo entre o consumidor e o produto; por outro, essa informação só é decifrada por um público mais específico, com maior conhecimento em relação a ingredientes utilizados ou a outro idioma.

Redução de peso e controle de patologias foram motivos pouco apontados para a leitura, não corroborando com estudo $^{15}$ que mostra que $70 \%$ dos usuários tem o hábito de ler a rotulagem portarem patologias que demandam modificações dietéticas e/ou seleção de alimentos específicos. Os consumidores, neste trabalho, relataram na entrevista que leem os rótulos dos alimentos com o objetivo de manter uma dieta saudável, ratificando achados em outro estudo ${ }^{14}$, o qual evidenciou que $76,4 \%$ dos indivíduos entrevistados afirmam que buscam as informações nos rótulos, pois desejam fazer escolhas saudáveis, priorizando a saúde como principal razão. Mostra-se, portanto, que existem diferentes prioridades na leitura dos rótulos nas diferentes populações estudadas.

Em relação às informações mais procuradas nos rótulos dos alimentos, o presente estudo se iguala a diversas pesquisas ${ }^{3,14,15,21}$, pois os itens mais destacados foram o valor calórico, quantidade de sódio e gorduras, mostrando uma relação entre o objetivo que os leva a leitura da rotulagem nutricional (alimentação saudável) e a informação mais utilizada.

Os meios de comunicação tiveram papel fundamental na busca de informações para a escolha e construção de hábitos alimentares, destacando-se no presente estudo a internet. Meios de comunicação como televisão, livros, jornais e revistas são importantes meios de fonte de informação, além do profissional nutricionista ${ }^{15}$, citado por uma a cada quatro pessoas entrevistadas neste trabalho.

Diversos critérios foram citados pelos consumidores para a escolha de determinados alimentos, com diferentes graus de importância, destacando-se qualidade e sabor. Esta avaliação pode fazer com que as empresas busquem melhorar seus produtos nos diferentes critérios considerados importantes pelos consumidores e utilizados no momento da compra, tais como preço e qualidade ${ }^{16}$, além do valor nutricional ${ }^{18}$.

Os rótulos dos alimentos devem ter uma linguagem clara para que não ocorram distorções e interpretações erradas, a fim de promoverem uma maior compreensão por parte da população ${ }^{20}$. Entretanto, a declaração nutricional não se apresenta legível no rótulo para $44,1 \%$ dos entrevistados e apenas 3\% dos consumidores compreendem totalmente as informações nutricionais apresentadas ${ }^{3}$. O presente trabalho pode ser comparado aos resultados de estudo semelhante ${ }^{15}$ onde a maioria dos consumidores diz encontrar as informações nutricionais, porém, os mesmos dizem não ter conhecimento suficiente sobre Nutrição para fazer escolhas certas na alimentação.

O grande desafio dos consumidores parece ser, então, compreender as informações e utilizá-las de forma adequada. Fatores como não saber informações corretas para a compreensão do assunto; não confiar na declaração nutricional e o fato de que o rótulo não é legível, leva consumidores a não verificar a informação nutricional além de desmotivar a leitura ${ }^{3}$.

Algumas medidas de intervenção citadas por consumidores poderiam ser utilizadas para que a declaração nutricional passasse a ser mais bem compreendida, entre elas: orientação sobre a declaração nutricional realizada por 
profissionais qualificados nos supermercados, por parte do estabelecimento e do fabricante dos produtos; divulgação na mídia sobre o que é a informação nutricional e sua importância e finalidade; a declaração nutricional poderia se apresentar de forma mais legível e em destaque, na parte da frente do produto; a linguagem apresentada nos rótulos deveria ser mais acessível, popular e simples; a veracidade da declaração nutricional apresentada nos rótulos pelo fabricante deveria ser verificada e comprovada por órgãos de fiscalização ${ }^{3}$.

Os dados obtidos em uma pesquisa ${ }^{20}$ mostraram que um dos problemas em relação ao conteúdo dos rótulos de alimentos é a falta de confiança na informação: os consumidores acreditam que as informações contidas ali são manipuladas pelas próprias empresas, interessadas em lucrar, e que estas informações não sofrem fiscalização nenhuma. Percebe-se, assim, a importância do desenvolvimento de políticas na área da educação e comunicação por parte das empresas, para que o consumidor possa compreender o que o rótulo informa.

Algumas limitações do estudo devem ser consideradas. Não foi realizada identificação da intenção de compra dos consumidores, nem do consumo alimentar dos entrevistados. Deste modo, a relação entre a declaração nutricional e a escolha de alimentos mais saudáveis foi estabelecida baseada na declaração dos consumidores de que a rotulagem nutricional influencia nas escolhas alimentares e na frequência dos elementos nutricionais identificados como indicadores de alimento saudável para o consumidor (calorias, sódio, gordura, etc). Sem dúvidas, os rótulos representam um meio de informação ao consumidor. Quando bem compreendidos, podem servir como ferramenta para serem adotadas escolhas alimentares saudáveis.

Pode-se concluir, com este estudo, que os consumidores possuem o hábito de leitura dos rótulos dos alimentos, e isto ocorre ainda no estabelecimento comercial, durante a compra. Nota-se que quanto maior a idade, o nível de escolaridade e a renda mensal, maior é este hábito, principalmente entre mulheres. Portanto, é de fundamental importância a realização de outros estudos, que busquem conhecer o comportamento dos consumidores em relação às escolhas alimentares, através da rotulagem dos alimentos, podendo assim criar políticas públicas para a habilitação dos consumidores.

\section{REFERÊNCIAS}

1. Pontes TE, Costa TF, Marum ABRF, Brasil ALD, Taddei JAAC. Orientação nutricional de crianças e adolescentes e os novos padrões de consumo: propagandas, embalagens e rótulos. Rev Paul Pediatr. 2009;27(1):99-105. https://doi.org/10.1590/S010305822009000100015
2. Nascimento CS. Validação de um instrumento de avaliação da compreensão da rotulagem nutricional pelo consumidor [monografia]. Brasília: Universidade de Brasília; 2004.

3. Souza SMFC, Lima KC, Miranda HF, Cavalcantil FID. Utilização da informação nutricional de rótulos por consumidores de Natal, Brasil. Rev Panam Salud Publ. 2011;29(5):337-43.

4. Carvalho JLV, Dias PDF, Oliveira AT, Amorim E. Orientação para rotulagem de alimentos. São Paulo: ABIMA/EMBRAPA; 2006.

5. Brasil. Resolução RDC 360, de 23 de dezembro de 2003. Regulamento técnico sobre rotulagem nutricional de alimentos embalados, tornando obrigatório a rotulagem nutricional [Internet]. DOU. 2003 Dez [citado em 2013 Maio 31]. Disponível em: http:// portal.anvisa.gov.br/wps/wcm/connect/1c2998004bc50d62a671 ffbc0f9:d5b29/RDC N 360 DE 23 DE DEZEMBRO DE 2003. pdf?MOD=AJPERES

6. Lobanco CM, Vedovato GM, Cano CB, Bastos DHM. Fidedignidade de rótulos de alimentos comercializados no município de São Paulo, SP. Rev Saúde Pública. 2009;43(3):499-505. https://doi. org/10.1590/S0034-89102009000300014

7. Segat ETL, Alves MK. Análise da veracidade da informação referente ao teor de vitamina $\mathrm{C}$ contida no rótulo de sucos de laranja industrializados e preparados sólidos para refresco. Nutr Pauta. 2012;11:27-30.

8. Camara MCC. Análise crítica da rotulagem de alimentos Diet e Light no Brasil [dissertação]. Rio de Janeiro: Fundação Oswald Cruz/ Escola Nacional de Saúde Pública Sérgio Arouca; 2007.

9. Garcia PPC, Carvalho LPS. Análise da rotulagem nutricional de alimentos Diet e Light. Ensaios e C. 2011;15(4):89-103.

10. Álvares F, Araújo WM, Borgo LA, Barros LM. Informações nutricionais em rótulos de queijos industrializados. Hig Aliment. $2005 ; 19(131): 25-33$.

11. Leonardi DS, Feres MBC, Portari GV, Jordão AA. Determinação do valor energético de hambúrgueres e almôndegas através da calorimetria direta e da composição centesimal. Comparação com informações nutricionais apresentadas nas embalagens. Biosci J. 2009;25(5):141-8.

12. Takahashi AA, Abreu ES. Avaliação da fidedignidade de informações nutricionais de bentos comercializados em um bairro oriental de São Paulo - SP. In: VIII Jornada da Iniciação Cientifica da Universidade Presbiteriana Mackenzie. São Paulo; 2011.

13. Sauerbronn ALA. Análise laboratorial da composição de alimentos processados como contribuição ao estudo da rotulagem nutricional obrigatória de alimentos e bebidas embalados no Brasil [dissertação] Rio de Janeiro: INCQS/FIOCRUZ; 2003.

14. Silva MZT. Influência da rotulagem nutricional sobre o consumidor [dissertação]. Recife: Universidade Federal de Pernambuco; 2003.

15. Cassemiro IA, Colauto NB, Linde GA. Rotulagem nutricional: quem lê e por quê? Arq Ciên Saúde Unipar. 2006;10(1):9-16.

16. Machado SS, Santos FO, Albinati FL, Santos LPR. Comportamento dos consumidores com relação à leitura de rótulos de produtos alimentícios. Alim Nutr. 2006;17(1):97-103

17. Cavada GS, Paiva FF, Helbig E, Borges LR. Rotulagem nutricional: você sabe o que está comendo? Braz J Food Technol. 2012; IV SSA:84-88. 
18. Pinheiro FA, Cardoso WS, Chaves KF, Oliveira ASB, Rios AS. Perfil de consumidores em relação à qualidade de alimentos e hábitos de compras. UNOPAR Cient Ciênc Biol Saúde. 2011;13(2): 95-102.

19. Cavada GS, Zanella R, Demoliner F, Orlandi S, Borges LR. Os consumidores leem os rótulos dos alimentos que consomem? Um estudo preliminar. In: Anais do XX Congresso de Iniciação Científica e III Mostra Científica da UFPEL. Pelotas; 2011.
20. Marins BR, Jacob SC, Peres F. Avaliação qualitativa do hábito de leitura e entendimento: recepção das informações de produtos alimentícios. Ciên Tecnol Aliment. 2008;28(3):579-85. https://doi. org/10.1590/S0101-20612008000300012

21. Monteiro RA, Coutinho JG, Recine E. Consulta aos rótulos de alimentos e bebidas por frequentadores de supermercados em Brasília, Brasil. Rev Panam Salud Publ. 2005;18(3):172-7. https:// doi.org/10.1590/S1020-49892005000800004 